\title{
Technique of percutaneous supraclavicular subclavian intra-aortic balloon pump placement
}

Tae H. Song, MD, and Valluvan Jeevanandam, MD

Feature Editor's Introduction-In the accompanying article, a technique is described for percutaneous insertion of an intra-aortic balloon pump (IABP) in the supraclavicular subclavian artery. Patients requiring mechanical circulatory support are often deconditioned due to preexisting illness, and their debilitation is worsened by immobility secondary to transfemoral device placement. Ambulation is possible with devices inserted through the subclavian and axillary arteries. This group from the University of Chicago highlights important technical considerations that improve the stability and safety of percutaneous subclavian IABP placement. They discuss issues such as insertion site preference, how to avoid device migration and kinking, and optimal techniques for device removal. Subclavian IABP insertion is unlikely to be the first choice for emergency IABP placement because it requires an experienced operator and imaging during placement, yet it may be a good option under less urgent conditions when the duration of support is anticipated to last several days or even several weeks. Our readers will benefit from lessons learned by the University of Chicago group, who recently reported their experience with 39 percutaneous placements in bridge to transplant patients. The ability to place these devices percutaneously, rather than through surgical axillary grafts, is a particularly appealing advance.

\section{Leora B. Balsam, MD}

Currently published techniques (surgical and percutaneous) have described intra-aortic balloon pump (IABP) placement in the axillary artery. ${ }^{1-3}$ The incidence of migration, driveline kinking, and IABP malfunction has been reported as a common problem (more than $40 \%$ in some series). ${ }^{2}$ Here we describe our preferred IABP placement technique, via the left supraclavicular subclavian artery, using a long wire-reinforced introducer. The straighter course

From the Section of Cardiac Surgery, Department of Surgery, University of Chicago Medicine, Chicago, Ill.

Received for publication March 22, 2021; accepted for publication March 29, 2021; available ahead of print May 11, 2021.

Address for reprints: Tae H. Song, MD, Section of Cardiac Surgery, Department of Surgery, University of Chicago Medicine, 5841 S Maryland Ave, E500A, MC5040, Chicago, IL 60637 (E-mail: thsong@uchicago.edu).

JTCVS Techniques 2021;8:20-2

2666-2507

Copyright (C) 2021 The Author(s). Published by Elsevier Inc. on behalf of The American Association for Thoracic Surgery. This is an open access article under the CC BY-NC-ND license (http://creativecommons.org/licenses/by-nc-nd/4.0/).

https://doi.org/10.1016/j.xjtc.2021.03.039 


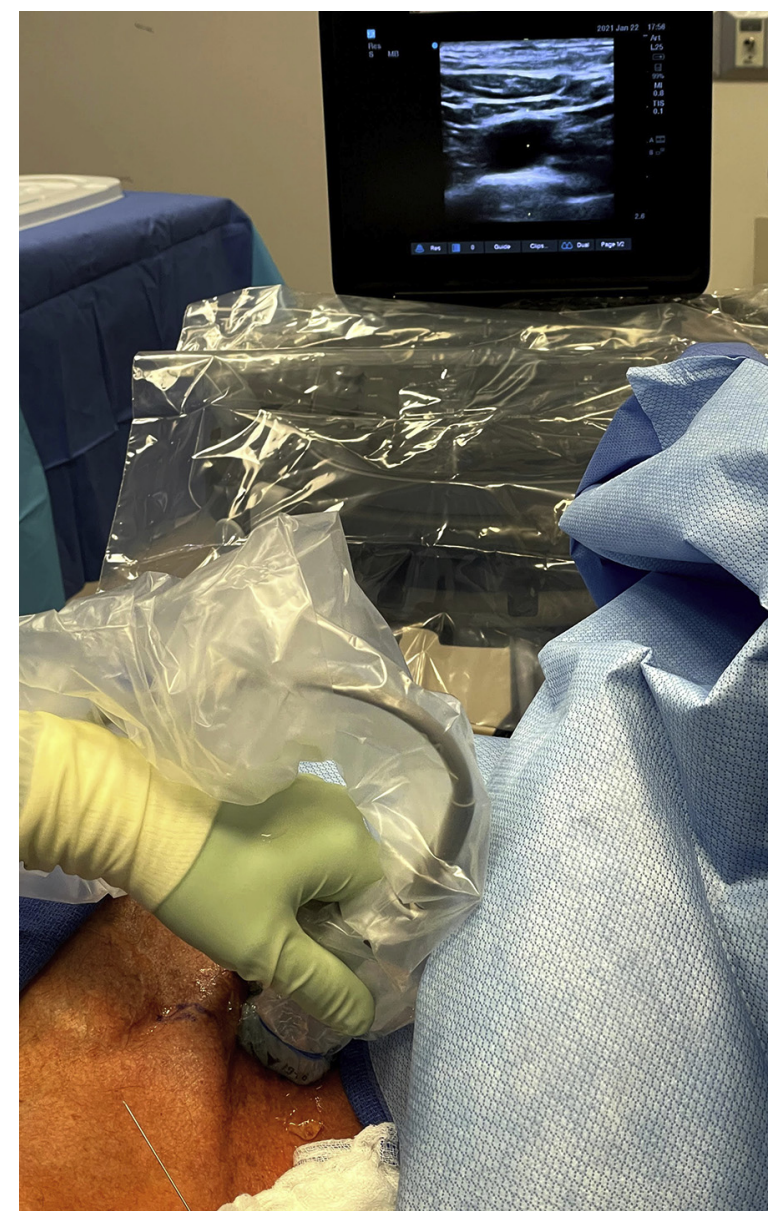

FIGURE 1. Visualization of the left supraclavicular subclavian artery on ultrasound.

well as the supraclavicular and infraclavicular areas, are prepped and draped in sterile manner. The cephalad drape should be positioned as posteriorly to the patient as possible (ie, close to the operating table) because access to the supraclavicular subclavian artery will sometimes require a posterior-to-anterior trajectory. The left side is used in nearly all cases to minimize the risk of cerebrovascular events. The supraclavicular approach is preferred due to the straighter course to the descending aorta. If removal of a femoral IABP is planned, the groin and IABP insertion site is also prepped and draped. The ultrasound probe is placed in a sterile cover containing ultrasound transmission gel and secured. Local anesthesia is administered, and percutaneous access is obtained. Use of micropuncture technique is strongly recommended, especially because the subclavian artery tends to be much more fragile than the femoral artery.

\section{IABP Placement}

Once access has been obtained and the micropuncture sheath has been placed, a digital subtraction angiography is performed, using $10 \mathrm{~mL}$ of a $50 \%$ water-soluble contrast. It is important to note the size of the artery, course to the aortic arch and descending aorta, branches near the access site, areas of stenosis, and presence of calcifications. This also helps determine whether percutaneous vascular closure in the future is feasible.

Then, a standard 0.035-in J-tip guidewire is advanced through the micropuncture sheath. The wire may advance to the descending aorta or the ascending aorta. The micropuncture sheath is then removed leaving the guidewire in place, and a 24-cm wire-reinforced 9F introducer is advanced (Arrow Super-Flex; Teleflex, Wayne, Pa). The wire-reinforced introducer stabilizes the IABP driveline after placement and reduces the incidence of IABP kinking and migration.

If the guidewire has been advanced to the descending aorta, the introducer is advanced until the tip is positioned at the level of the lower border of the left mainstem bronchus. If the wire is located in the ascending aorta, the introducer is advanced until it is within the aortic arch, and a modified right coronary angiography catheter (AR MOD; Cordis, Hialeah, Fla) is used to redirect the guidewire to the descending aorta and then the introducer is advanced.

Once the introducer is in appropriate position in the descending aorta, the guidewire is changed to the 0.025-in IABP guidewire. The tip of the wire is confirmed to be in the aortoiliac system with fluoroscopy. Then, the IABP is advanced over the guidewire and positioned so that the proximal marker is approximately $2 \mathrm{~cm}$ beyond the tip of the wire-reinforced sheath (Figure 2). The IABP is then connected to the console and counterpulsation is started. If a femoral IABP is in place, it is kept on until the introducer and IABP guidewire is in place, then turned off and pulled down while the subclavian IABP is advanced and activated.

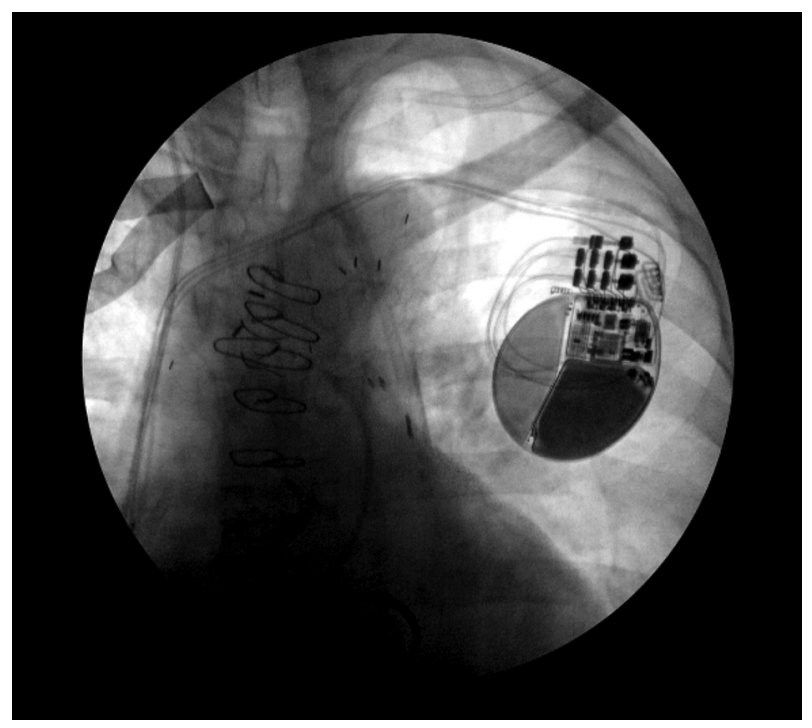

FIGURE 2. Positioning of the wire-reinforced introducer and subclavian intra-aortic balloon pump. 


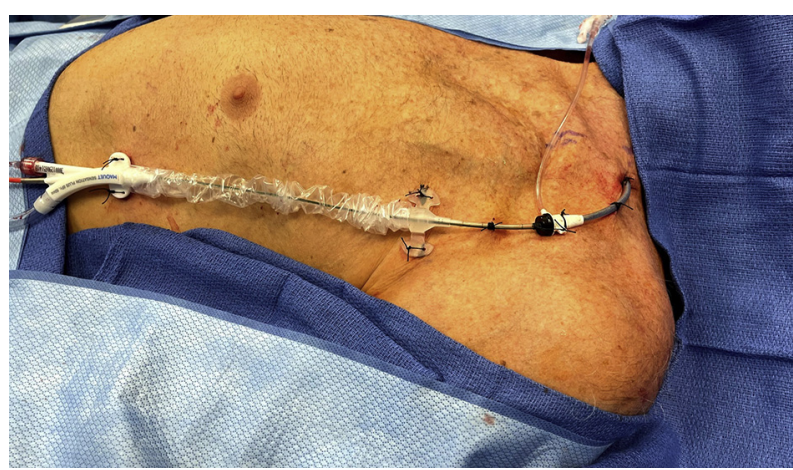

FIGURE 3. Securing the subclavian intra-aortic balloon pump.

It is important to adequately secure the subclavian IABP because the primary goal of this IABP placement technique is to allow ambulation and rehabilitation. Finally, 0 -silk sutures are used to secure the sheath and the IABP tabs, but the IABP driveline itself is also secured to the skin as well as the sheath (Figure 3). Adhesive film such as Ioban (3M, Saint Paul, Minn) can be used to secure the IABP and sheath further.

\section{Removal}

Subclavian and axillary artery IABPs are removed using an Angio-Seal device (Terumo, Tokyo, Japan) for percutaneous vascular closure when the artery is larger than $5 \mathrm{~mm}$, with no branches noted in proximity to the access site on angiography at time of placement. The removal procedure is performed in the intensive care unit. The IABP insertion site is prepped and draped, then the IABP is cut close to the introducer, and an 0.025-in guidewire is advanced through the central lumen. The IABP and introducer are removed over the wire, and an 8F Angio-Seal device is advanced and deployed. If an Angio-Seal cannot be used, the IABP and sheath are removed and hemostasis is achieved with manual compression. The supraclavicular subclavian artery is easier to compress than the axillary artery, and also avoids proximity to the brachial plexus and related complications.

\section{CONCLUSIONS}

Percutaneous subclavian IABPs can be used to provide counterpulsation support while allowing patients to ambulate and participate in physical therapy. Mechanical circulatory support is being used with increasing frequency as a means to bridge patients to heart transplant, durable device implant, or recovery. ECMO and other forms of temporary support are also used but carry a higher morbidity and complication rate. IABP placement via the supraclavicular subclavian artery, with added stability using a wirereinforced introducer, is a mechanical circulatory support option that can be applied to various clinical scenarios.

\section{Conflict of Interest Statement}

The authors reported no conflicts of interest.

The Journal policy requires editors and reviewers to disclose conflicts of interest and to decline handling or reviewing manuscripts for which they may have a conflict of interest. The editors and reviewers of this article have no conflicts of interest.

\section{References}

1. Russo MJ, Jeevanandam V, Stepney J, Merlo A, Johnson EM, Malyala R, et al. Intra-aortic balloon pump inserted through the subclavian artery: a minimally invasive approach to mechanical support in the ambulatory end-stage heart failure patient. J Thorac Cardiovasc Surg. 2012;144:951-5.

2. Estep JD, Cordero-Reyes AM, Bhimaraj A, Trachtenberg B, Khalil N, Loebe M, et al. Percutaneous placement of an intra-aortic balloon pump in the left axillary/ subclavian position provides safe, ambulatory long-term support as bridge to heart transplantation. JACC Heart Fail. 2013;1:382-8.

3. Rosenbaum AN, Jain CC, Shadrin IY, El Hajj SC, El Sabbagh A, Behfar A. Percutaneous axillary intra-aortic balloon pump insertion technique as bridge to advanced heart failure therapy. ASAIO J. 2021;67:e81-5.

Key Words: intra-aortic balloon pump, percutaneous, ambulatory, subclavian, axillary 